\title{
Directed Acceleration of Electrons from a Solid Surface by Sub-10-fs Laser Pulses
}

\author{
F. Brandl, ${ }^{1}$ B. Hidding, ${ }^{1}$ J. Osterholz,${ }^{1}$ D. Hemmers, ${ }^{1}$ A. Karmakar, ${ }^{2}$ A. Pukhov, ${ }^{2}$ and G. Pretzler ${ }^{1}$ \\ ${ }^{1}$ Institut für Laser- und Plasmaphysik, Heinrich-Heine-Universität Düsseldorf, 40225 Düsseldorf, Germany \\ ${ }^{2}$ Institut für Theoretische Physik I, Heinrich-Heine-Universität Düsseldorf, 40225 Düsseldorf, Germany
}

(Received 17 August 2007; published 11 May 2009)

\begin{abstract}
Electrons have been accelerated from solid target surfaces by sub-10-fs laser pulses of $120 \mu \mathrm{J}$ energy which were focused to an intensity of $2 \times 10^{16} \mathrm{~W} / \mathrm{cm}^{2}$. The electrons have a narrow angular distribution, and their observed energies exceed $150 \mathrm{keV}$. We show that these energies are not to be attributed to collective plasma effects but are mainly gained directly via repeated acceleration in the transient field pattern created by incident and reflected laser, alternating with phase-shift-generating scattering events in the solid.
\end{abstract}

PACS numbers: 52.38.Kd, 41.75.Jv, 52.35.Mw

Laser-based electron acceleration has been a rapidly evolving topic through the last decade. Well-directed multi-100-MeV electron bunches can be generated indirectly via excitation of longitudinal plasma waves in underdense plasmas with relativistic laser pulses [1]. With lasersolid interaction, broad-cone emission beams moving into the forward direction through the overdense target are obtained, being heavily influenced by collisions [2]. This is important in connection with proton and ion acceleration [3] and fast ignition [4], for example. Sometimes, confined electron beams have also been observed pointing away from the target on the laser side [5-8], all of the latter with laser pulse energies of a few to a few hundreds of $\mathrm{mJ}$ showing a strong dependence on the properties of the expanding plasma on the solid surface.

Another type of laser is used in many labs with much lower pulse energies $(\leq 1 \mathrm{~mJ})$, but durations less than $10 \mathrm{fs}$ and repetition rates around $1 \mathrm{kHz}$. When focused onto a solid surface, such lasers can produce plasma of near-solid density and peak temperatures above $100 \mathrm{eV}$, mainly due to the absence of significant preplasma and the ultrashort pulse duration [9]. In this Letter, we show that such laser pulses with only $120 \mu \mathrm{J}$ of energy can be used for directed electron acceleration off solid surfaces with energies well above $100 \mathrm{keV}$. The process is much more deterministic compared to previous experiments, since it is dominated by the interaction of the light fields with free electrons together with collisions in the solid, whereas the plasma plays no significant role. The experimental results give insight to the detailed field structure at the surface and to absorption of the laser pulse.

A commercial Ti:Sa CPA laser system similar to that described in [10] was used in the experiments. The obtained 20-fs laser pulses were spectrally broadened in a Nefilled hollow fiber and compressed by a set of chirped mirrors [11] to a final pulse length of 9 fs (FWHM). Pulses were focused with an $f / 3$ off-axis parabola to a spot size of $3.5 \mu \mathrm{m}$ (FWHM). With a pulse energy of $120 \mu \mathrm{J}$ in the focal spot, the average intensity (within the range where $I \geq I_{\max } / e^{2}$ both temporally and spatially) was $2 \times 10^{16} \mathrm{~W} / \mathrm{cm}^{2}$. The intensity contrast was $>10^{5}$ up to 1 ps before the pulse, which confined the preplasma to a range smaller than the skin layer as confirmed by spectroscopic measurements with the same laser [9].

In the experiments, $p$-polarized pulses hit the target under $45^{\circ}$ in single shot mode. Isolators like glass and boron nitride $(\mathrm{BN})$ and different metals $(\mathrm{Al}, \mathrm{Ag}$, and $\mathrm{Au}$; $Z=13,47,79$ ) were used as targets, mostly with opticalquality flat surfaces. The detection of fast electrons was performed by imaging plates (IPs, type FUJI). IPs allow for absolute measurements of the electron numbers, if the energy distribution is known [12]. Two diagnostics were employed (Fig. 1): First, the angular electron distribution was recorded by placing an IP at a distance of $\sim 13 \mathrm{~cm}$ from the target (see Fig. 2). Second, an electron spectrometer was set up consisting of a metallic aperture (diameter $1 \mathrm{~mm})$, two metallic plates $\left(4 \times 9 \mathrm{~cm}^{2}\right)$, of which one was on positive voltage (up to $5 \mathrm{kV}$ ), and an IP. The $E$ field and Earth's magnetic field created parabolic electron traces on

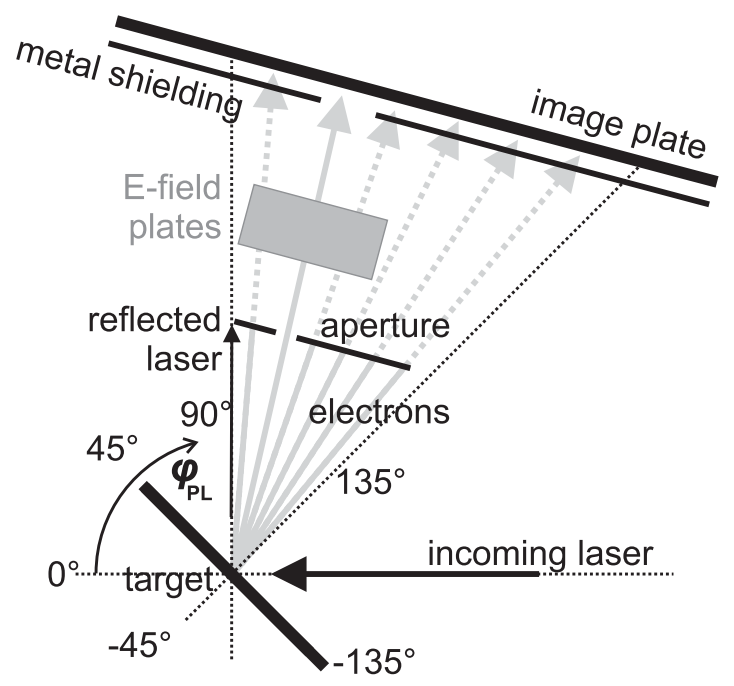

FIG. 1. Experimental setup for spectral measurements. Used without aperture, $E$ field and shielding for 2D survey images. 


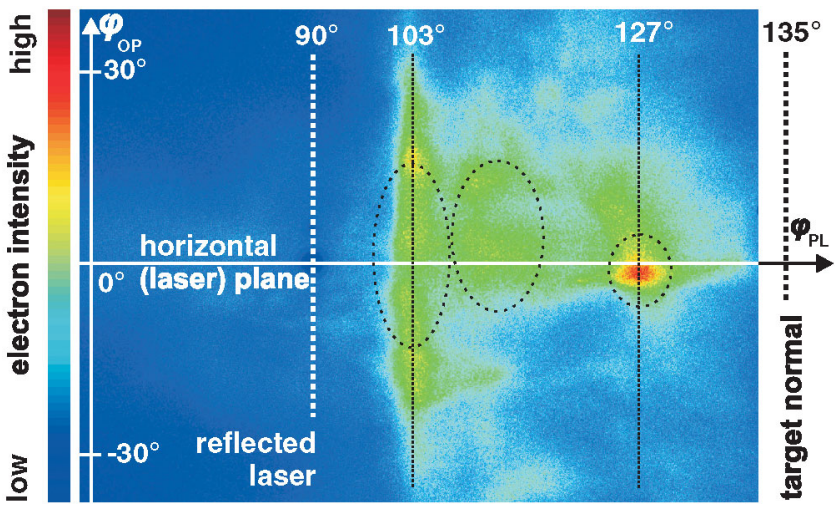

FIG. 2 (color). Typical 2D survey image of electrons detected with an IP for a single laser shot on an Al target. Emission is predominantly at distinct angles (highlighted regions) between target normal and the direction of the reflected laser.

the detector IP (see inset in Fig. 3). The electrons were tracked using CST Particle Studio [13].

The first result was that electrons emitted from the interaction region run to distinct directions (Fig. 2). They formed one or several confined spots on the IP, typically around the horizontal laser plane and at angles between 100 and $130^{\circ}$. This pattern was reproducible from shot to shot and similar with all investigated metals. The use of isolators as target material (e.g., glass substrates) strongly reduced the amount of accelerated electrons, and unpolished surfaces led to a reduction of directionality.

Electron spectra were measured at such angles where strong emission was found. Maximum energies above $150 \mathrm{keV}$ were detected (Fig. 3) with all metal targets. For the purpose of comparison, Maxwellian distribution functions were fitted to the data with temperature parameters in the range of $20 \mathrm{keV}$. For isolators, these temperatures were only slightly lower (e.g., $17 \mathrm{keV}$ for $\mathrm{BN}$ ), but the numbers of detected particles per laser shot were much smaller (ranging from above 6000 for $\mathrm{Al}$ to $\sim 2000$ for $\mathrm{Au}$ as compared to $\sim 200$ for BN). Typically, energies were higher at

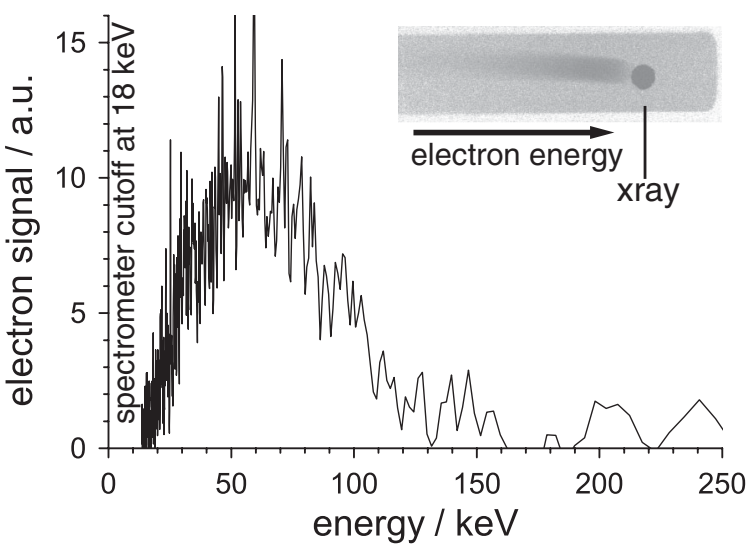

FIG. 3. Electron spectrum obtained close to the direction of the reflected laser for a single laser shot on a silver target. The inset shows raw data as recorded on the IP. angles closer to the reflected laser direction $\left(\sim 105^{\circ}\right)$ and decreased towards the target normal direction. With the spectral information and the angular distribution, the number of electrons detected in survey images was estimated to $3 \times 10^{7}$ per shot.

The measured electron energies are far too high for ponderomotive acceleration in the laser focus, the ponderomotive potential amounting to $U_{p}=4.7 \mathrm{keV}$, only. Furthermore, the very steep plasma gradient [9] rules out collective plasma effects like resonance absorption to be dominant. To confirm this, a 3D simulation of the experiment has been performed using the Virtual Laser Plasma Lab (VLPL) PIC code [14]. A $p$-polarized laser pulse of wavelength $\lambda=0.82 \mu \mathrm{m}$ hit an overdense plasma layer under $45^{\circ}$ with spot radius $R=1.64 \mu \mathrm{m}$, duration $T=9 \mathrm{fs}$, and a dimensionless light amplitude $a_{0}=0.2$. The plasma density rose sharply from 0 to 50 times critical within $0.1 \mu \mathrm{m}$, reflecting a scale length as seen in previous experiments [9]. The simulation box of size $3 \lambda \times 10 \lambda \times 1 \lambda$ was sampled with a grid of $120 \times 100 \times 2$ cells with up to 1000 particles per cell.

VLPL has been equipped with binary collisions. The energy dependent scattering cross section $\sigma$ for electrons with atoms (in this case, aluminum) was taken from literature [15] to obtain the scattering probability. Figure 4 compares the energy spectrum and angular distribution of the hot electrons leaving the front surface to the results when collisions are switched off. Obviously, collisionless plasma effects (grey curve in Fig. 4) are insufficient to reproduce the experimental results, and an interplay of particle acceleration in the laser field and collisions within the dense plasma is required.

For a more detailed analysis, trajectories of single electrons during acceleration were investigated by two separate Monte Carlo simulations, namely, in the vacuum and within solid-density matter. To begin with, we studied the interaction of electrons with the dynamic interference structure generated by the overlap of the incoming and

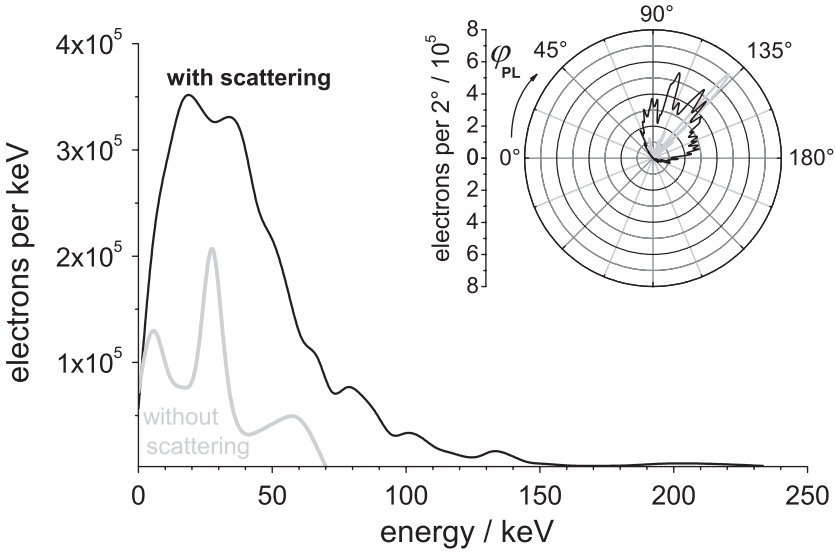

FIG. 4. Electron spectra and angular distributions (inset) obtained by PIC simulations with and without scattering. The experimental energies $\geq 70 \mathrm{keV}$ can only be reproduced with scattering. Angles are defined as in Fig. 1. 
reflected light pulses in the focus. The electromagnetic field of the laser was modeled analytically under the following assumptions: First, we did not take into account the softening of the surface by creation and expansion of a plasma because this effect is too slow to play a significant role for light propagation as long as the 9-fs laser pulse is on. This is justified by observations presented in [9] showing that for very similar conditions, the plasma scale length at the time of the pulse maximum is $2.9 \mathrm{~nm}$. Second, the surface was treated like a metal in the sense that a phase jump of $\pi$ was assumed for the reflected beam. Third, we assumed laser absorption to take place within the skin layer of the target such that the intensity of the reflected light pulse in vacuum was a fraction $r I_{0}$ of the incident intensity $I_{0}$.

Under these assumptions, infinite $p$-polarized plane waves lead to an interference field which is linearly polarized at the center of the target surface and points to the direction $\varphi=\arctan (-\sqrt{1 / r})$. The polarization and direction characteristics in front of the surface vary spatially with a periodicity of $\sqrt{2} \lambda / 2$. The field amplitude is enhanced by a factor of $\sqrt{1+r^{2}}$ when compared to the incoming field alone, being the well-known result of superposition of two coherent light fields. The interesting feature in the case of an ultrashort pulse is that the acceleration of electrons is determined by the temporal and spatial details of these field oscillations, and not-as usual - by an average over many oscillations.

We included longitudinal field components in addition to the dominant transverse components [16-18] for a precise description of the tightly focused pulses. Constant values of $r \approx 55 \%$ and $r \approx 25 \%$ were assumed in the simulations shown in Figs. 5 and 6, respectively. This brings out the important features of the experimental data and is motivated by intensity-dependent reflectivities [19].

For the results shown in Fig. 5, single electrons were emitted from the solid surface into the described field at different times with random energies in the $\mathrm{eV}$ range, and their trajectories were calculated by the relativistic equation of motion. Dependent on emission time, these electrons are accelerated away from the target or back into it, and they gain up to $23 \mathrm{keV}$ [see Fig. 5(a)], which is of the order of their maximum oscillation energy in the field. The emission of electrons above $10 \mathrm{keV}$ is well directed within a cone of $<5 \mathrm{msr}$ to a direction close to that of the interference field at the surface [a reflectivity of 55\% corresponds to angles of $\sim-55^{\circ}$ into the target and $\sim 125^{\circ}$ off the target as shown in Fig. 5(b)], being in good agreement with the measured collimated feature at $\sim 127^{\circ}$ in Fig. 2. This part of the acceleration process is similar to Brunel heating [20], but here outgoing electrons are considered and not those accelerated into the target.

With this model, the observed electron emission directions can be explained, the injection time and reflectivity determining the direction of the outgoing electrons. However, the maximum energies are an order of magnitude
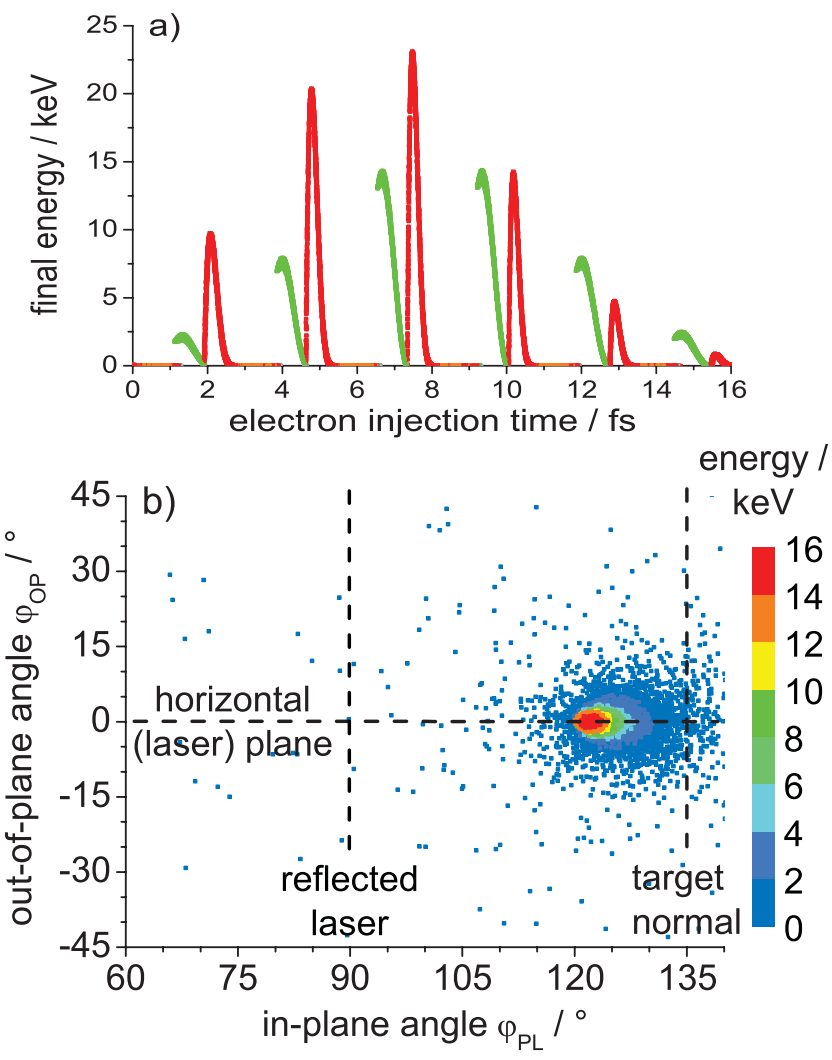

FIG. 5 (color). Acceleration of electrons (initial energies $\leq$ $20 \mathrm{eV}$ ) off a solid surface by a sub-10-fs laser pulse incident under $45^{\circ}$. (a) The laser phase at the emission time determines final energy as well as direction. Electrons are either reinjected into the target within one laser cycle (red) or accelerated away from it (green). (b) The direction is mainly determined by the field direction at the target surface (here $r=55 \%$ ). Only the particles emitted away from the surface are shown.

higher than calculated, which demands an extension of the model. From the variety of collisional absorption mechanisms, it is well known that trajectories of electrons can be substantially altered by collisions in the skin layer and beyond, sometimes even accompanied with energy gain as with inverse bremsstrahlung, for example. Apart from energy shift, such (multiple) scattering events can lead to reemission of preaccelerated electrons into the transient laser field in the vacuum outside the solid surface, where they can receive a further accelerating push under certain circumstances. For example, our simulations show that $50 \mathrm{keV}$ electrons can gain another $50 \mathrm{keV}$ in the laser interference field when leaving the target, for instance, or up to $35 \mathrm{keV}$ when driven back to the target. Note that the whole acceleration in front of the target surface is not a statistical process (in contrast to the scattering inside the target) but collision-free and deterministic: The dynamics of an electron is fully described by its momentum as well as injection time and location when leaving the solid.

The paths of electrons in the solid were simulated with the second MC code, which treats scattering at the bulk atoms according to the cross sections of [15]. Bethe-Bloch- 


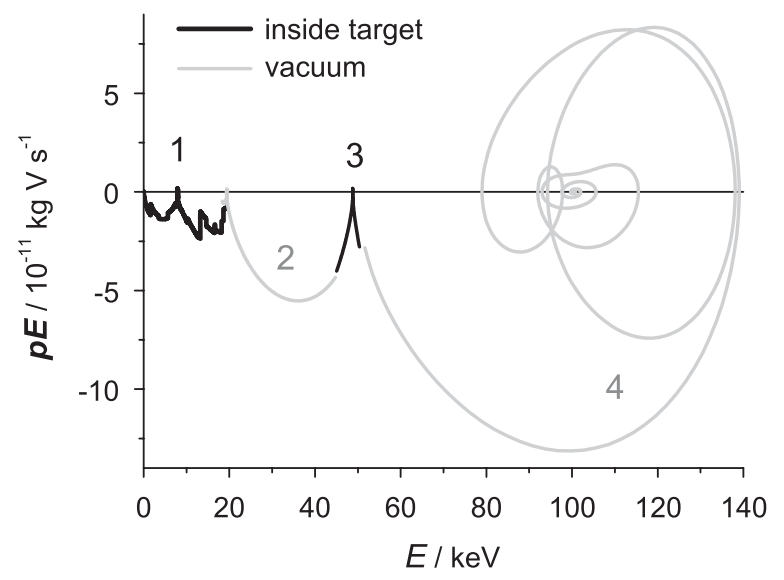

FIG. 6. Scalar product of momentum and electric field vector vs kinetic energy along the path of a moving test particle typical for the case of strong acceleration. The particle is extracted from within the target (1), accelerated and driven back into it (2) and scattered back into the vacuum (3). Finally, it receives a strong boost of kinetic energy on its complex way out of the region of the laser field (4).

type energy loss was included as well as the influence of the laser field declining within the skin depth $(\sim 15 \mathrm{~nm})$. It was found that indeed a considerable fraction of the electrons accelerated into the solid are scattered in such a way that they move inside during the decelerating phase of the laser field and are reemitted into the next accelerating phase. In principle, this can happen several times during the laser pulse interaction, albeit with decreasing probability as scattering cross sections decrease with higher electron energies.

Figure 6 shows the total history of a test particle which gains an energy of $\sim 100 \mathrm{keV}$. The interplay of scattering inside the target (paths 1 and 3) and deterministic acceleration in the interference field in vacuum (paths 2 and 4) leads to multiple harvesting of the optimum phase of acceleration in the laser field, thus multiplying the final energy by a factor of about 7 compared to the maximum one-path acceleration (Fig. 5).

The absolute number of electrons measured in the hot population is roughly matched when all the conduction band electrons in the focal spot region are considered. Ionization by the laser field and by collisions adds further electrons, but only those set free early enough in the laser pulse can be accelerated to high energies.

The conditions for absorption and specular reflection varying on the fs scale lead to a changing interference field and thus to different acceleration directions for the different laser cycles. It is therefore probable that each of the dominant emission directions contains electrons finally ejected in a certain laser cycle, thus sampling the specular reflectivity cycle by cycle. The less energetic electrons emitted at larger angles (e.g., feature at $127^{\circ}$ in Fig. 2) have most likely been accelerated directly without additional scattering and reemission and are thus well colli- mated. The faster electrons at smaller angles exhibit less collimation (e.g., see feature at $103^{\circ}$ in $\varphi_{\mathrm{OP}}$ direction) due to deleterious scattering effects.

The weak dependence of the electron energies on the atomic number of the metal targets originates from a balance of two effects. On the one hand, low- $Z$ materials allow efficient extraction of low-energy electrons from within the skin layer due to small energy losses, leading to higher starting populations, whereas on the other hand, backscattering of high energy electrons works much better with high- $Z$ materials. In isolators, the lack of initially quasifree electrons explains their strongly reduced efficiency, and with rough surfaces, the spatial structure of the interference field and thus the directionality of acceleration is disturbed. A highly nonlinear dependence on laser intensity was found (i.e., at half intensity the integrated electron signal dropped by more than a factor of 20), which is another indication for the necessity of successive acceleration stages.

In conclusion, directed electron ejection off solid surfaces was demonstrated using laser intensities of about $2 \times$ $10^{16} \mathrm{~W} / \mathrm{cm}^{2}$ and few-cycle pulse durations below $10 \mathrm{fs}$. In contrast to longer laser pulses, it is concluded that plasma effects are of minor importance for the acceleration of highest energy electrons. The interaction is governed by the interaction of the light field with single electrons, leading to distinct ejection angles. The unexpectedly high energies observed are explained by a repeated interplay of acceleration in vacuum and scattering inside the solid which leads to phase-delayed reemission into the field. This way, the kinetic energies are boosted by accelerating an electron in subsequent laser pulse cycles.

This work has been supported by the DFG programs TR 18 and GRK 1203.

[1] E. Esarey and C. B. Schroeder, LBNL Report No. LBNL53510, 2003.

[2] P. Gibbon, Phys. Rev. E 72, 026411 (2005).

[3] S. C. Wilks et al., Phys. Plasmas 8, 542 (2001).

[4] M. H. Key, Phys. Plasmas 14, 055502 (2007).

[5] S. Bastiani et al., Phys. Rev. E 56, 7179 (1997).

[6] L. M. Chen et al., Phys. Rev. Lett. 87, 225001 (2001).

[7] Y. T. Li et al., Phys. Rev. Lett. 96, 165003 (2006).

[8] X. H. Yuan et al., Phys. Plasmas 15, 013106 (2008).

[9] J. Osterholz et al., Phys. Rev. Lett. 96, 085002 (2006).

[10] M. Hentschel et al., Appl. Phys. B 70, Suppl. 1, 161 (2000).

[11] Z. Cheng et al., Opt. Lett. 24, 247 (1999).

[12] K. A. Tanaka et al., Rev. Sci. Instrum. 76, 013507 (2005).

[13] http://www.cst.com/content/products/ps/overview.aspx.

[14] A. Pukhov, J. Plasma Phys. 61, 425 (1999).

[15] M. Riley et al., At. Data Nucl. Data Tables 15, 443 (1975).

[16] B. Quesnel and P. Mora, Phys. Rev. E 58, 3719 (1998).

[17] M. Lax et al., Phys. Rev. A 11, 1365 (1975).

[18] L. Cicchitelli, H. Hora, and R. Postle, Phys. Rev. A 41, 3727 (1990).

[19] H. M. Milchberg et al., Phys. Rev. Lett. 61, 2364 (1988).

[20] F. Brunel, Phys. Rev. Lett. 59, 52 (1987). 